\title{
Retraction: Decoupling Interrupts from the Internet in Markov Models
}

\author{
Jinwen Ma, Jingchun Zhang, and Jinrong Guo
}

School of Information Science and Engineering, Lanzhou University, Lanzhou 730000, China

e-mail: guojr11@lzu.edu.cn

Several conference proceedings have been infiltrated by fake submissions generated by the SCIgen computer program. Due to the fictional content the chapter "Decoupling Interrupts from the Internet in Markov Models" by "Jinwen Ma, Jingchun Zhang, and Jinrong Guo" has been retracted by the publisher. Measures are being taken to avoid similar breaches in the future. 